\title{
Solvation Structure and Thermodynamics of Oxyanions from Dielectric Relaxation Spectroscopy and Molecular Simulation
}

\author{
BENJAMIN GILBERT ${ }^{1}$, PIOTR ZARZYCKI ${ }^{2}$ AND \\ CHRISTOPHER COLLA ${ }^{3}$ \\ ${ }^{1}$ Lawrence Berkeley National Lab \\ ${ }^{2}$ Lawrence Berkeley National Laboratory \\ ${ }^{3}$ Lawrence Livermore National Laboratory \\ Presenting Author: bgilbert@lbl.gov
}

Many biogeochemically important elements including nutrients (e.g., phosphorus, sulfur) and essential metals (e.g., molybdenum, selenium) are found in natural waters as oxyanions. The speciation, transport and reactivity of oxyanions, as for all solutes in water, are strongly affected by the hydration free energy and the structure and dynamics of the waters of hydration. The establishment of robust molecular understanding for the solvation of ions is a longstanding challenge in aqueous geochemistry. Solvation theory provides a coherent description of the enthalpic and entropic contributions to the hydration of simple hydrophilic or hydrophobic solutes. However, the contributions to solvation from both water-solute and waterwater hydrogen bonding in the hydration shell appear to be important but difficult to assess directly. Dielectric relaxation spectroscopy (DRS) measures the rotational mobility of dipolar molecules, such as water, and can distinguish water molecules in solution and at surfaces with altered mobility due to different hydrogen-bond configurations. We present the DRS studies, complemented by molecular dynamics simulation that provide new insights into the hydration shells of carbonate, nitrate, silicate ions and related molecules. The hydration shells are composed of water molecules that are either immobilized or that exhibit slower relaxation dynamics than bulk water. Estimates of the total hydration number reveal significant variation with oxyanion speciation, indicating the strong role that the oxyanion oxygen molecules play as hydrogen-bond acceptors and the center of the acidity/negative charge of the ion. The hydration shells show strong dependence on temperature, with a non-linear reduction of hydration number with increasing temperature. By assuming that water association to oxyanions can be characterized by a chemical equilibrium constant, analysis of the temperature-dependent data reveals a strong contribution of water-water hydrogen-bond interactions to oxyanion solvation. 